\title{
Differential dissipativity theory for dominance analysis
}

\author{
Fulvio Forni, Rodolphe Sepulchre
}

\begin{abstract}
High-dimensional systems that have a lowdimensional dominant behavior allow for model reduction and simplified analysis. We use differential analysis to formalize this important concept in a nonlinear setting. We show that dominance can be studied through linear dissipation inequalities and an interconnection theory that closely mimics the classical analysis of stability by means of dissipativity theory. In this approach, stability is seen as the particular situation where the dominant behavior is 0 -dimensional. The generalization opens novel tractable avenues to study multistability through 1-dominance and limit cycle oscillations through 2-dominance.
\end{abstract}

\section{INTRODUCTION}

The analysis of a system is considerably simplified when it is low-dimensional. Linear system analysis frequently exploits the property that a few dominant poles capture the main properties of a possibly high-dimensional system. Lowdimensional models are even more critical in nonlinear system analysis. Multistability or limit cycle analysis is difficult beyond the phase plane analysis of two-dimensional systems.

In this paper we seek to formalize the property that a nonlinear system has low-dimensional dominant behavior. Our approach is differential: we characterize the property for linear systems and then study the nonlinear system differentially, that is, along the linearized flow in the tangent bundle. The seminal example of differential analysis in control theory is contraction analysis [28], [36], [16], [39], [45], which we interpret as a differential analysis of exponential stability. The property is the contraction of a ball, characterized via a Lyapunov dissipation inequality. A nonlinear system is contractive when this dissipation inequality holds infinitesimally along any of its trajectories. In the present paper, the corresponding property is 0-dominance: it ensures that the dominant behavior of the nonlinear system is 0-dimensional. A more recent example of differential analysis is differential positivity [13], the differential analysis of positivity. The linear property is the contraction of a cone, also characterized by a dissipation inequality. A nonlinear system is differentially positive when this dissipation inequality holds infinitesimally along any of its trajectories. In the context of the present paper, the corresponding property is 1-dominance: it guarantees that the dominant behavior of the nonlinear system is 1-dimensional. More generally, we study the property of $p$-dominance differentially. The property

F. Forni and R. Sepulchre are with the University of Cambridge, Department of Engineering, Trumpington Street, Cambridge CB2 1PZ f.fornileng.cam.ac.uk|r.sepulchredeng.cam.ac.uk. The research leading to these results has received funding from the European Research Council under the Advanced ERC Grant Agreement Switchlet n.670645. is characterized via a dissipation inequality, which is then required to hold infinitesimally along the trajectories of the nonlinear system. We prove a general theorem that formalizes that the dominant behavior of a $p$-dominant system is $p$ dimensional.

Differential analysis is general in that it allows to extend a linear system property to an arbitrary flow defined on a differentiable manifold. An important restriction in this paper is that we only consider dissipation properties characterized by linear matrix inequalities. Furthermore, we impose the linear dissipation inequality to be uniform on the tangent bundle. In geometric terms, this endows the differentiable manifold with a flat non-degenerate metric structure, characterized by a constant quadratic form.

The linear-quadratic restriction allows for a fruitful bridge with the linear-quadratic theory of dissipativity. Many of the available computational tools of dissipativity theory become available for the analysis of $p$-dominance. The analysis of $p$-dominance is reformulated as the search of a quadratic differential storage that decreases along the solutions of the linearized dynamics. The classical interconnections theorems of (linear-quadratic) dissipativity theory are reformulated as tools that facilitate that construction. The only substantial difference with the classical theory is that the quadratic form that characterizes the storage is no longer required to be a Lyapunov function, i.e. positive definite. Instead, it is required to have a fixed inertia, that is, $p$ negative eigenvalues and $n-p$ positive eigenvalues. Stability corresponds to $p=0$, whereas $p$-dominance allows for any integer $0 \leq p \leq n$.

The paper is organized as follows. Section II characterizes $p$-dominance and $p$-dissipativity for linear time-invariant models. Section III defines $p$-dominance for nonlinear systems with two main results that characterize their asymptotic behavior. The connection with related results in the literature is discussed in Section $\mathrm{V}$ and dominance analysis via the solution of LMIs is illustrated in Section VI Differential $p$-dissipativity is addressed in Section VII, where we primarily illustrate the role of differential passivity and differential small-gain theorems in the design and analysis of multistable systems $(p=1)$ and limit cycle oscillations $(p=2)$. The proofs of the main theorems are provided in appendix.

\section{DOMInANT LTI SYSTEMS}

Definition 1: A linear system $\dot{x}=A x$ is $p$-dominant with rate $\lambda \geq 0$ if there exists a symmetric matrix $P$ with inertia $(p, 0, n-p)$ such that

$$
A^{T} P+P A \leq-2 \lambda P-\varepsilon I .
$$


for some $\varepsilon \geq 0$. The property is strict if $\varepsilon>0$.

We recall that a matrix with inertia $(p, 0, n-p)$ has $p$ negative eigenvalues, and $n-p$ positive eigenvalues. For simplicity in what follows we will use the abbreviated terminology inertia $p$ to denote those matrices.

In terms of the quadratic form $V(x)=x^{T} P x$, the (strict) dissipation inequality (1) reads

$$
\begin{aligned}
\dot{V}(x) & =x^{T}\left(A^{T} P+P A\right) x \\
& \leq-2 \lambda V(x)-\varepsilon|x|^{2}
\end{aligned}
$$

For $\varepsilon>0$ this implies that the two cones

$$
\mathcal{K}^{-}=\left\{x \in \mathbb{R}^{n} \mid V(x) \leq 0\right\}, \mathcal{K}^{+}=\left\{x \in \mathbb{R}^{n} \mid V(x) \geq 0\right\}
$$

are strictly contracting either in forward or in backward time:

$$
\forall t>0: e^{-A t} \mathcal{K}^{+} \subset \mathcal{K}^{+}, e^{A t} \mathcal{K}^{-} \subset \mathcal{K}^{-}
$$

Equivalent characterizations of $p$-dominance are provided in the following proposition, whose proof is in the preliminary version of this paper [14].

Proposition 1: For $\varepsilon>0$, the Linear Matrix Inequality (1) is equivalent to any of the following conditions:

1) The matrix $A+\lambda I$ has $p$ eigenvalues with strictly positive real part and $n-p$ eigenvalues with strictly negative real part.

2) there exists an invariant subspace splitting $\mathbb{R}^{n}=\mathcal{H} \oplus \mathcal{V}$ such that $A \mathcal{H} \subset \mathcal{H}$ and $A \mathcal{V} \subset \mathcal{V}$. The dimension of $\mathcal{V}$ is $n-p$ and the dimension of $\mathcal{H}$ is $p$. Furthermore, there exist constants $0<\underline{C} \leq 1 \leq \bar{C}$ and $\underline{\lambda}<\lambda<\bar{\lambda}$ such that

$$
\begin{aligned}
& \forall x \in \mathcal{H}:\left|e^{A t} x\right| \geq \underline{C} e^{-\underline{\lambda} t}|x|, t \geq 0 \\
& \forall x \in \mathcal{V}:\left|e^{A t} x\right| \leq \bar{C} e^{-\bar{\lambda} t}|x|, t \geq 0 .
\end{aligned}
$$

The property of $p$-dominance ensures a splitting between $n-p$ transient modes and $p$ dominant modes. Only the $p$ dominant modes dictate the asymptotic behavior. Because $\lambda \geq 0$ and $\mathcal{V} \subset \mathcal{K}^{+}$, the quadratic form $V(x)$ is a Lyapunov function for the transient behavior, that is, for the restriction of the flow in $\mathcal{V}$.

For $p=0, p$-dominance is the classical property of exponential stability: all modes are transient and the asymptotic behavior is 0-dimensional.

The matrix inequality [1] is equivalent to the conic constraint

$$
\left[\begin{array}{c}
\dot{x} \\
x
\end{array}\right]^{T}\left[\begin{array}{cc}
0 & P \\
P & 2 \lambda P+\varepsilon I
\end{array}\right]\left[\begin{array}{l}
\dot{x} \\
x
\end{array}\right] \leq 0 .
$$

Dissipativity theory extends $p$-dominance to open systems by augmenting the internal dissipation inequality with an external supply. The external property of $p$-dissipativity is captured by a conic constraint between the state of the system $x$, its derivative $\dot{x}$, and the external variables $y$ and $u$ of the form

$$
\left[\begin{array}{l}
\dot{x} \\
x
\end{array}\right]^{T}\left[\begin{array}{cc}
0 & P \\
P & 2 \lambda P+\varepsilon I
\end{array}\right]\left[\begin{array}{l}
\dot{x} \\
x
\end{array}\right] \leq\left[\begin{array}{l}
y \\
u
\end{array}\right]^{T}\left[\begin{array}{cc}
Q & L \\
L^{T} & R
\end{array}\right]\left[\begin{array}{l}
y \\
u
\end{array}\right]
$$

where $P$ is a matrix with inertia $p, \lambda \geq 0, L, Q, R$ are matrices of suitable dimension, and $\varepsilon \geq 0$. The property is strict if $\varepsilon>$
0 . We call supply rate $s(y, u):=y^{T} Q y+y^{T} L u+u^{T} L^{T} y+$ $u^{T} R u$ the right-hand side of $(3)$. An open dynamical system is $p$-dissipative with rate $\lambda$ if its dynamics $\dot{x}=A x+B u$, $y=C x+D u$ satisfy (3) for all $x$ and $u$. The property has a simple characterization in terms of linear matrix inequalities.

Proposition 2: A linear system $\dot{x}=A x+B u, y=C x+D u$ is $p$-dissipative with rate $\lambda \geq 0$ if and only if there exists a symmetric matrix $P$ with inertia $p$ such that

$$
\left[\begin{array}{cc}
A^{T} P+P A+2 \lambda P-C^{T} Q C+\varepsilon I & P B-C^{T} L-C^{T} Q D \\
B^{T} P-L^{T} C-D^{T} Q C & -D^{T} Q D-L^{T} D-D^{T} L-R
\end{array}\right] \leq 0 .
$$

Proof: $[\Rightarrow]$ Just replace $\dot{x}=A x+B u$ and $y=C x+D u$ in (3) and rearrange. [ $\Leftarrow$ ] Multiply (4) by $\left[x^{T} u^{T}\right]$ on the left, and by $\left[x^{T} u^{T}\right]^{T}$ on the right. Then we get $\dot{x}^{T} P x+x^{T} P \dot{x}+$ $2 \lambda x^{T} P x<s(y, u)$ as desired.

An interconnection theorem can be easily derived. A proof is provided in the preliminary version of this paper [14], see also the proof of Theorem 4

Proposition 3: Let $\Sigma_{1}$ and $\Sigma_{2} p_{1}$-dissipative and $p_{2}$ dissipative systems respectively, with uniform rate $\lambda$ and with supply rate

$$
s_{i}\left(y_{i}, u_{i}\right)=\left[\begin{array}{l}
y_{i} \\
u_{i}
\end{array}\right]^{T}\left[\begin{array}{ll}
Q_{i} & L_{i} \\
L_{i}^{T} & R_{i}
\end{array}\right]\left[\begin{array}{l}
y_{i} \\
u_{i}
\end{array}\right]
$$

for $i \in\{1,2\}$. The closed-loop system given by negative feedback interconnection

$$
u_{1}=-y_{2}+v_{1} \quad u_{2}=y_{1}+v_{2}
$$

is $\left(p_{1}+p_{2}\right)$-dissipative with rate $\lambda$ from $v=\left(v_{1}, v_{2}\right)$ to $y=$ $\left(y_{1}, y_{2}\right)$ with supply rate

$$
s(y, v)=\left[\begin{array}{l}
y \\
v
\end{array}\right]^{T}\left[\begin{array}{cc|cc}
Q_{1}+R_{2} & -L_{1}+L_{2}^{T} & L_{1} & R_{2} \\
-L_{1}^{T}+L_{2} & Q_{2}+R_{1} & -R_{1} & L_{2} \\
\hline L_{1}^{T} & -R_{1} & R_{1} & 0 \\
R_{2} & L_{2}^{T} & 0 & R_{2}
\end{array}\right]\left[\begin{array}{l}
y \\
v
\end{array}\right] .
$$

Furthermore, the closed-loop system is $\left(p_{1}+p_{2}\right)$-dominant with rate $\lambda$ if

$$
\left[\begin{array}{cc}
Q_{1}+R_{2} & -L_{1}+L_{2}^{T} \\
-L_{1}^{T}+L_{2} & Q_{2}+R_{1}
\end{array}\right] \leq 0
$$

Mimicking classical dissipativity theory, there are two important particular cases of supply rates : the passivity supply

$$
s(y, u)=\left[\begin{array}{l}
y \\
u
\end{array}\right]^{T}\left[\begin{array}{ll}
0 & I \\
I & 0
\end{array}\right]\left[\begin{array}{l}
y \\
u
\end{array}\right] .
$$

and the gain supply:

$$
s(y, u)=\left[\begin{array}{l}
y \\
u
\end{array}\right]^{T}\left[\begin{array}{cc}
-I & 0 \\
0 & \gamma^{2} I
\end{array}\right]\left[\begin{array}{l}
y \\
u
\end{array}\right]
$$

Hence, Proposition 3 provides an analog of the small-gain theorems and passivity theorems for $p$-dominance of a linear time-invariant system. 


\section{DifFERENTIAL ANALYSIS OF} DOMINANT NONLINEAR SYSTEMS

For a nonlinear system

$$
\dot{x}=f(x) \quad x \in \mathcal{X}
$$

we define dominance differentially, that is, through the linear dissipation inequality

$$
\left[\begin{array}{l}
\dot{\delta x} \\
\delta x
\end{array}\right]^{T}\left[\begin{array}{cc}
0 & P \\
P & 2 \lambda P+\varepsilon I
\end{array}\right]\left[\begin{array}{l}
\dot{\delta x} \\
\delta x
\end{array}\right] \leq 0
$$

for every $\delta x \in T_{x} \mathcal{X}$, where $P$ is a matrix with inertia $p$ and $\varepsilon \geq 0$.

In what follows we assume that $\mathcal{X}$ is a smooth Riemannian manifold of dimension $n$. Given any $\delta x \in T_{x} \mathcal{X},|\delta x|$ denotes the Riemannian metric on $\mathcal{X}$ represented by $\sqrt{\delta x^{T} \delta x}$ in local coordinates. $\psi^{t}(x)$ denotes the flow of (11) at time $t$ passing through $x \in \mathcal{X}$ at time $0 . \partial \psi^{t}(x)$ denotes the differential of $\psi^{t}(x)$ with respect to $x$. Note that $\left(\psi^{t}(x), \partial \psi^{t}(x) \delta x\right)$ is a flow in the tangent bundle. It is the solution of the prolonged system [7]

$$
\left\{\begin{aligned}
\dot{x} & =f(x) \\
\dot{\delta x} & =\partial f(x) \delta x \quad(x, \delta x) \in T \mathcal{X}
\end{aligned}\right.
$$

at time $t$ passing through $(x, \delta x) \in T \mathcal{X}$ at time 0 . In local coordinates $\partial f(x)$ denotes the differential of $f$ at $x$ and $P$ is the local representation of a metric tensor $P$ with fixed inertia.

Following the approach of Section III, we define the property of dominance for nonlinear systems as follows.

Definition 2: A nonlinear system $\dot{x}=f(x)$ is $p$-dominant with rate $\lambda \geq 0$ if there exists a symmetric matrix $P$ with inertia $p$ such that $(12)$ is satisfied by the solutions of the prolonged system 13 for some $\varepsilon \geq 0$. The property is strict if $\varepsilon>0$.

In terms of the quadratic function $V(\delta x):=\delta x^{T} P \delta x$, the differential dissipation inequality 12 reads

$$
\begin{aligned}
\dot{V}(\delta x) & =\delta x^{T}\left(\partial f(x)^{T} P+P \partial f(x)\right) \delta x \\
& \leq-2 \lambda V(\delta x)-\varepsilon|\delta x|^{2}
\end{aligned}
$$

As a consequence, for $\varepsilon>0$, the two cone fields

$$
\begin{array}{r}
\mathcal{K}^{+}(x):=\left\{\delta x \in T_{x} \mathcal{X} \mid V(\delta x) \geq 0\right\} \\
\mathcal{K}^{-}(x):=\left\{\delta x \in T_{x} \mathcal{X} \mid V(\delta x) \leq 0\right\}
\end{array}
$$

are strictly contracting either in forward time or in backward time, respectively:

$$
\begin{array}{rr}
\partial \psi^{-t}(x) \mathcal{K}^{+}(x) \subset \mathcal{K}^{+}\left(\psi^{-t}(x)\right) & \forall t>0 \\
\partial \psi^{t}(x) \mathcal{K}^{-}(x) \subset \mathcal{K}^{-}\left(\psi^{t}(x)\right) & \forall t>0
\end{array}
$$

The following result provides the differential analog of Proposition 1 .

Theorem 1: Let $\mathcal{A} \subseteq \mathcal{X}$ be a compact invariant set and let (11) be a strictly $p$-dominant system with rate $\lambda \geq 0$ and tensor $P$. Then, for each $x \in \mathcal{A}$, there exists an invariant splitting $T_{x} \mathcal{X}=\mathcal{H}_{x} \oplus \mathcal{V}_{x}$ such that

$$
\begin{array}{rlrl}
\partial \psi^{t}(x) \mathcal{H}_{x} & \subseteq \mathcal{H}_{\psi^{t}(x)} & \forall t & \in \mathbb{R}, \\
\partial \psi^{t}(x) \mathcal{V}_{x} \subseteq \mathcal{V}_{\psi^{t}(x)} & \forall t & \in \mathbb{R} .
\end{array}
$$

$\mathcal{H}_{x}$ and $\mathcal{V}_{x}$ are distributions of dimension $p$ and $n-p$ respectively. Furthermore, there exist constants $\underline{C} \leq 1 \leq \bar{C}$ and $\underline{\lambda}<\lambda<\bar{\lambda}$ such that

$$
\begin{array}{ll}
\left|\partial \psi^{t}(x) \delta x\right| \geq \underline{C} e^{-\underline{\lambda} t}|\delta x| & \forall x \in \mathcal{A}, \forall \delta x \in \mathcal{H}_{x} \\
\left|\partial \psi^{t}(x) \delta x\right| \leq \bar{C} e^{-\bar{\lambda} t}|\delta x| & \forall x \in \mathcal{A}, \forall \delta x \in \mathcal{V}_{x} .
\end{array}
$$

The interpretation of the theorem is that the linearized flow $\partial \psi^{t}(\cdot)$ admits an invariant splitting between $n-p$ transient modes and $p$ dominant modes. The $p$ dominant modes dictate the long-term behavior of the flow. The quadratic form $V(\delta x)$ is a differential Lyapunov function in the invariant distribution $\mathcal{V} \subset T \mathcal{X}$

For $\mathcal{X}=\mathbb{R}^{n}$ the characterization of the asymptotic behavior of dominant systems can be further refined. This is because, by integration, the differential dissipation inequality (14) leads to the incremental inequality

$$
\begin{aligned}
\dot{V}(x-y) & =(x-y)^{T} P(f(x)-f(y))+(f(x)-f(y))^{T} P(x-y) \\
& =2(x-y)\left(\int_{0}^{1} P \partial f(s x+(1-s) y) d s\right)(x-y) \\
& \leq-\varepsilon(x-y)^{T}\left(\int_{0}^{1}-2 \lambda P+\varepsilon I d s\right)(x-y) \\
& \leq-2 \lambda V(x-y)-\varepsilon|x-y|^{2} .
\end{aligned}
$$

The following result is based on the incremental dissipation inequality $(17)$. We denote by $\Omega(x)$ the $\omega$-limit set of $x$, that is, the set of all $\omega$-limit points of $x$.

Theorem 2: For $\mathcal{X}=\mathbb{R}^{n}$, let $(11)$ be a strictly $p$-dominant system with rate $\lambda \geq 0$. Then, the flow on any compact $\omega$ limit set is topologically equivalent to a flow on a compact invariant set of a Lipschitz system in $\mathbb{R}^{p}$.

For small values of $p$, Theorem 2 severely constrains the possible attractors of the system.

Corollary 1: Under the assumptions of Theorem 2, every bounded solution asymptotically converges to

- a unique fixed point if $p=0$;

- a fixed point if $p=1$;

- a simple attractor if $p=2$, that is, a fixed point, a set of fixed points and connecting arcs, or a limit cycle.

\section{Proofs of TheOrems 1 And 2}

Proof of Theorem 1

Invariant splitting. For any $p>0$ and for any $\delta x \in \mathcal{K}^{-}$, the dissipation inequality (14) implies

$$
\dot{V}(\delta x) \leq-2 \lambda V(\delta x)-\varepsilon|\delta x|^{2} \leq-\varepsilon|\delta x|^{2}
$$

for all $x \in \mathcal{X}$ and all $\delta x$ on the boundary of $\mathcal{K}^{-}$, which guarantees that

$$
\begin{aligned}
& \forall t \geq 0: \partial \psi^{t} \mathcal{K}^{-} \subseteq \mathcal{K}^{-} \\
& \forall t>0: \partial \psi^{t}\left(\mathcal{K}^{-} \backslash\{0\}\right) \subset \mathcal{K}^{-} .
\end{aligned}
$$

The dissipation inequality (14) also implies

$$
\dot{V}(\delta x) \leq-2 \lambda V(\delta x)-\varepsilon|\delta x|^{2} \leq-\left(2 \lambda-\varepsilon_{1}\right) V(\delta x)
$$

for $\varepsilon_{1}:=\frac{\varepsilon}{\left|\lambda_{\min }(P)\right|}>0$. Time-integration of this inequality yields the estimate

$$
\forall t \geq 0: \frac{e^{2 \lambda t} V\left(\partial \psi^{t} \delta x\right)}{V(\delta x)} \geq e^{\varepsilon_{1} t}
$$


which holds uniformly for all $x \in \mathcal{X}$ and all $\delta x$ in the interior of $\mathcal{K}^{-}$. (19) and (21) guarantee that there exist $T>0$ and $\mu>1$ such that $\frac{\left|e^{\lambda t} \partial \psi^{t} \delta x\right|}{|\delta x|} \geq \mu$ for all $t \geq T$, all $x \in \mathcal{X}$ and all $\delta x \in \mathcal{K}^{-}$.

Likewise, for any $n-p>0$ and for any $\delta x \in \mathcal{K}^{+}$, the dissipation inequality (14) implies

$$
\dot{V}(\delta x) \leq-2 \lambda V(\delta x)-\varepsilon|\delta x|^{2} \leq-\left(2 \lambda+\varepsilon_{2}\right) V(\delta x)
$$

for $\varepsilon_{2}=\frac{\varepsilon}{\lambda_{\max }(P)}>0$. Integration of the first inequality backward time guarantees that

$$
\begin{aligned}
& \forall t \geq 0: \partial \psi^{-t} \mathcal{K}^{+} \subseteq \mathcal{K}^{+} \\
& \forall t>0: \partial \psi^{-t}\left(\mathcal{K}^{+} \backslash\{0\}\right) \subset \mathcal{K}^{+} .
\end{aligned}
$$

Integration of the second inequality backward time also yields the estimate

$$
\forall t \geq 0: \frac{e^{-2 \lambda t} V\left(\partial \psi^{-t} \delta x\right)}{V(\delta x)} \geq e^{\varepsilon_{2} t}
$$

which holds uniformly for all $x \in \mathcal{X}$ and all $\delta x$ in the interior of $\mathcal{K}^{+}$. As above, (23) and (24) guarantee that there exist $T>0$ and $\mu>1$ such that $\frac{\left|e^{-\lambda t} \partial \psi^{-t} \delta x\right|}{|\delta x|} \geq \mu$ for all $t \geq T$, all $x \in \mathcal{X}$ and all $\delta x \in \mathcal{K}^{+}$.

From here, we proceed as in the proof of [35, Theorem 1.2] (see also [2, Chapter 3]) to show that

$$
\begin{aligned}
\mathcal{H}_{x} & :=\bigcap_{t \geq 0} e^{\lambda t} \partial \psi^{t}(x) \mathcal{K}^{-}\left(\psi^{-t}(x)\right) \subset \mathcal{K}^{-}(x) \\
\mathcal{V}_{x} & :=\bigcap_{t \geq 0} e^{-\lambda t} \partial \psi^{-t}\left(\psi^{t}(x)\right) \mathcal{K}^{+}\left(\psi^{t}(x)\right) \subset \mathcal{K}^{+}(x)
\end{aligned}
$$

are invariant distributions of dimension $p$ and $n-p$ respectively, that is,

$$
\begin{aligned}
e^{\lambda t} \partial \psi^{t}(x) \mathcal{H}_{x} & \subseteq \mathcal{H}_{\psi^{t}(x)} & \forall t & \in \mathbb{R}, \\
e^{\lambda t} \partial \psi^{t}(x) \mathcal{V}_{x} & \subseteq \mathcal{V}_{\psi^{t}(x)} & \forall t & \in \mathbb{R} .
\end{aligned}
$$

Since $e^{\lambda t}$ is just a scalar factor, (15) follows.

Exponential estimates. Observe that $\delta x \in \mathcal{H}$ implies that $\delta x$ belongs to the interior of $\mathcal{K}^{-}$. The estimate (16a) with $\underline{\lambda}=\lambda-\frac{\varepsilon_{1}}{2}$ follows from the fact that $-V(\delta x)$ is positive definite in $\mathcal{H}$ and that $V(\delta x)$ satisfies 20). For instance, there exist $0<\rho_{1} \leq \rho_{2}$ such that $\rho_{1} \delta x^{T} \delta x \leq-V(\delta x) \leq \rho_{2} \delta x^{T} \delta x$ for all $\delta x \in \overline{\mathcal{H}}$ and (20) gives $\rho_{2}\left|\partial \psi^{t} \delta x\right|^{2} \geq-V\left(\partial \psi^{t} \delta x\right) \geq$ $-e^{-\left(2 \lambda-\varepsilon_{1}\right) t} V(\delta x) \geq e^{-\left(2 \lambda-\varepsilon_{1}\right) t} \rho_{1}|\delta x|^{2}$ for all $t \geq 0$, from which (16a) follows.

Likewise, $\delta x \in \mathcal{V}$ implies that $\delta x$ belongs to the interior of $\mathcal{K}^{+}$. The estimate $(16 \mathrm{~b})$ with $\bar{\lambda}=\lambda+\frac{\varepsilon_{2}}{2}$ follows from the fact that $V(\delta x)$ is positive definite in $\mathcal{V}$ and satisfies 22.

Proof of Theorem 2. From the dissipation inequality (17) we derive the inequality $\frac{d}{d t} e^{2 \lambda t} V(x-y)=e^{2 \lambda t} \dot{V}+2 \lambda e^{2 \lambda t} V(x-$ $y) \leq-e^{2 \lambda t} \varepsilon|x-y|^{2}$ which, by time integration, implies the following estimate for any pair of solutions initialized at $x_{0}, y_{0} \in \mathcal{X}$ :

$$
\begin{array}{r}
\forall t \geq 0: V\left(\psi^{t}\left(x_{0}\right)-\psi^{t}\left(y_{0}\right)\right) \leq e^{-2 \lambda t} V\left(x_{0}-y_{0}\right)- \\
-\varepsilon \int_{0}^{t} e^{2 \lambda(\tau-t)}\left|\psi^{\tau}\left(x_{0}\right)-\psi^{\tau}\left(y_{0}\right)\right|^{2} d \tau
\end{array}
$$

For large $t \geq 0$, the first term on the right hand side vanishes. This implies that the difference between any two solutions either asymptotically vanishes or eventually remains in the cone $\mathcal{K}^{-}$. We conclude that if $x$ and $y$ are distinct $\omega$-limit points, then necessarily $V(x-y)<0$.

Consider any compact set $\Omega$ of $\omega$-limit points. Let $\mathcal{H}_{P}$ and $\mathcal{V}_{P}$ the invariant subspaces of the matrix $P$ associated to the $p$ negative and $n-p$ positive eigenvalues, respectively. Define the linear projection $\Pi: \mathcal{X} \rightarrow \mathcal{H}_{P}$ parallel to $\mathcal{V}_{P}$. We claim that $\Pi$ restricted to $\Omega$ is one-to-one. This is because $x \neq y$ and $\Pi(x-y)=0$ imply $V(x-y)>0$, which was proved to contradict 25 .

The remaining argument follows the proof of [24, Theorem 3.17]. If $y \in \Pi \Omega(x)$ then $y=\Pi z$ for a unique $z \in \Omega(x)$ and the flow $\Pi \psi^{t}(z)$ on $\mathcal{H}_{P}$ is generated by the vector field

$$
F(y):=\Pi f\left(\Pi^{-1}(y)\right) \quad y \in \Omega(x),
$$

which is Lipschitz by construction.

\section{Connections With the Literature}

\section{A. Dominated splittings and dominance}

The property of dominance studied in this paper is closely related to the cousin concepts of dominated splittings and partial hyperbolicity. Both concepts have appeared in dynamical systems theory as part of the extensive research to generalize the key concept of hyperbolicity pioneered by Smale and Anosov in the 60's. The common theme of that research line is that robust features of smooth dynamical systems should be captured by robust features of their linear approximations. Robust is to be understood here in the sense of structural stability, that is, robustness to small perturbations of the vector field.

The subject is vast but we refer the interested reader to the recent survey [40] for an orientation map. Both dominated splittings and partial hyperbolicity continue to be an important subject in dynamical systems theory [8], [40], [38], [21], [26], [37]. We refer the reader to [53], [37] for the implications of Theorem 1 on normal hyperbolicity and structural stability of compact attractors. Dominated splittings have also received attention in control theory [6].

Theorem 1 and its proof are grounded in the results and proofs of [35, Theorem 1.2] and [13, Theorem 3]. Theorem 2 and its proof are grounded in the results and proofs of [24 Theorem 3.17] and [41, Proposition 3]. The proof merges these approaches with the techniques in [43].

The decomposition $T \mathcal{X}=\mathcal{H} \oplus \mathcal{V}$ in Theorem 1 is a splitting that is invariant under the linearized flow $\partial \psi^{t}$. The splitting is called dominated because the flow satisfies

$$
\frac{\left|\partial \psi^{t}(x) \delta x_{v}\right|}{\left|\partial \psi^{t}(x) \delta x_{h}\right|} \leq \frac{\bar{C}}{\underline{C}} e^{-(\bar{\lambda}-\underline{\lambda}) t} \frac{\left|\delta x_{v}\right|}{\left|\delta x_{h}\right|}
$$

for any $\delta x_{h} \neq 0 \in \mathcal{H}_{x}$ and $\delta x_{v} \in \mathcal{V}_{x}$. The dominated splitting is a consequence of the contraction of the cone fields $\mathcal{K}^{+}(x)$ and $\mathcal{K}^{-}(x)$.

The contraction of a cone is projective, that is, it expresses a contraction of the non-dominant directions relative to the dominant directions of the flow. Because of the assumption of a nonnegative dissipation rate $\lambda \geq 0$, dominance further imposes vertical contraction, that is, contraction of the flow 
$\partial \psi^{t}$ in the vertical distribution $\mathcal{V}$, see $(16 \mathrm{~b})$. The requirement of vertical contraction is an extra requirement of dominance with respect to the property of dominated splitting. Theorem 2 does not hold without this extra requirement.

\section{B. Contraction, differential stability, and 0-dominance}

A strict 0-dominant system is a contractive system [28], [36], [39], [12]. For a linear system, the property is simply exponential stability, meaning hyperbolicity and contraction of the $n$ transient modes to the 0-dimensional attractor. Because $P$ is positive definite, the dissipation inequality implies the contraction of an ellipsoid. The quadratic form $V(\delta x)$ is a differential Lyapunov function in the terminology of [12]. On vector spaces $\mathcal{X}=\mathbb{R}^{n}$, its integration along geodesic curves leads to the incremental Lyapunov function $V(x-y)$. From (17), it implies exponential contraction of the difference between any two trajectories. The attractor of a 0-dominant system is necessarily a unique fixed point.

\section{Monotonicity, differential positivity, and 1-dominance}

Strictly 1-dominant systems are strictly differentially positive systems [13], [10]. The contractive cone $\mathcal{K}^{-}$is an ellipsoidal cone, that is, it is the union of two solid pointed convex cones $\mathcal{K}^{-}=-\mathcal{K}^{*} \cup \mathcal{K}^{*}$ as illustrated in Figure 1

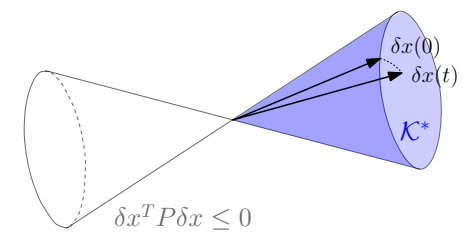

Figure 1. For $\varepsilon>0$ 12 guarantees that any trajectory moves from the boundary of $\mathcal{K}^{*}$ towards the interior.

A linear system that contracts a solid pointed convex cone is a positive system [29], [5]. Positivity with respect to an ellipsoidal cone was characterized via LMIs in [46], [47]. See also [18] for a recent use of such characterization in model reduction. Differential positivity thus induces a dominated splitting between one dominant direction and $n-1$ dominated directions. The reader is referred to [13], [10] for a comprehensive analysis of the asymptotic behavior of differentially positive systems.

The distinction between 1-dominance and differential positivity is again the property of projective contraction versus vertical contraction. Differential positivity does not imply contraction in the 1-dimensional vertical subspace $\mathcal{V}_{x}$. As a consequence, the statement of Corollary 1 for $p=1$ only holds for generic initial conditions of strictly differentially positive systems, see [13, Corollary 5].

For $\mathcal{X}=\mathbb{R}^{n}, 1$-dominance is also tightly related to monotonicity [42], [23], [1], [24]. A monotone system preserves a partial order $\preceq$ : any pair of trajectories $x(\cdot), y(\cdot)$ from ordered initial conditions $x(0) \preceq y(0)$ satisfy $x(t) \preceq y(t)$ for all $t \geq 0$.

Monotonicity is implied by 1-dominance. The partial order is the usual partial order associated to a pointed convex cone: $x \preceq y$ iff $y-x \in \mathcal{K}^{*}$. Monotonicity requires that trajectories $y(0)-x(0) \in \mathcal{K}^{*}$ satisfy $y(t)-x(t) \in \mathcal{K}^{*}$ for all $t \geq 0$, which is guaranteed by 1 -dominance by the invariance $V(x(t)-y(t)) \leq 0$ for all $t \geq 0$ and by the fact that if $x(0)-y(0)=0$ then $x(t)-y(t)=0$, for all $t \geq 0$. Here also, monotonicity is independent of $\lambda$. The assumption $\lambda \geq 0$ makes 1-dominance stronger than monotonicity and the statement of Corollary 1 for $p=1$ only holds for generic initial conditions of monotone systems, see [20], [24].

\section{Contraction of rank 2 cones and 2-dominance}

The property of 2-dominance provides the following generalization of Poincaré-Bendixson theorem:

Corollary 2: For $p=2$, under the assumptions of Theorem 2. let $\mathcal{U} \subseteq \mathcal{X}$ be a compact forward invariant set that does not contain fixed points. Then, the $\omega$-limit set of any point in $\mathcal{U}$ is a closed orbit.

Proof: Take any $\omega$-limit set contained in $\mathcal{U}$. By Theorem 2 the flow restricted to this set is topologically equivalent to the flow of a planar system. By Poincaré-Bendixson theorem [22. Chapter 11, Section 4], a nonempty compact limit set of a planar system which contains no fixed points is a closed orbit.

A similar generalization was developed in the papers [43], [44], [41] by generalizing the concept of monotonicity to rank2 cones. It is this generalization that motivated the results in the present paper. Note that the statement of Corollary 2 only holds for generic initial conditions of rank 2 monotone systems. The stronger conclusion of Corollary 2 is again due to the assumption of a nonnegative dissipation rate $\lambda \geq 0$.

\section{E. Invariant cone fields}

The assumption of a constant matrix $P$ makes the cone fields $\mathcal{K}^{+}(x)$ and $\mathcal{K}^{-}(x)$ constant, that is, the same cone is attached to every $x \in \mathcal{X}$. A more intrinsic characterization for $\mathcal{X}=\mathbb{R}^{n}$ is that the cone field is invariant by translation, the natural group action on a vector space. This geometric interpretation allows for extensions on Lie groups and, more generally, homogeneous spaces [31], [32], [3]. The definition of dominance thus assumes an invariant cone field in the present paper. The invariance of the cone field is an important source of tractability for the search of the storage.

\section{Algorithmic TEST FOR $p$-DOMINANCE AND A SIMPLE EXAMPLE}

\section{A. Dominant spectral splitting}

A necessary condition for dominance is that the spectrum of the family of matrices $\partial f(x)+\lambda I, x \in \mathcal{X}$, admits a uniform splitting, see Figures 2 (left) and 3 (left) for an illustration.

Theorem 3: Let (11) be a strictly $p$-dominant system. Then, there exists a maximal interval $\left(\lambda_{\min }, \lambda_{\max }\right)$ such that $\partial f(x)+$ $\lambda I$ has $p$ unstable eigenvalues and $n-p$ stable eigenvalues (negative real part) for each $\lambda \in\left(\lambda_{\min }, \lambda_{\max }\right)$, for every $x \in$ $\mathcal{X}$.

Proof: By Proposition 1, the feasibility of $\partial f(x)^{T} P+$ $P \partial f(x)+2 \lambda P \leq-\varepsilon I$, for $\varepsilon>0$ and for $P$ of inertia $p$ guarantees that each matrix $\partial f(x)+\lambda I$ has $p$ unstable 
eigenvalues and $n-p$ stable eigenvalues (negative real part) at each $x \in \mathcal{X}$. The splitting of the eigenvalues of $\partial f(x)$ at each $x$ is preserved for every value of $\lambda$ within some given spectral gap $\left(\lambda_{\min }(x), \lambda_{\max }(x)\right)$. Thus, by the uniformity of the strict inequality above, $\lambda_{\min }:=\sup _{x \in \mathcal{X}} \lambda_{\min }(x)<\lambda$ and $\lambda_{\max }:=\inf _{x \in \mathcal{X}} \lambda_{\max }(x)>\lambda$.

Spectral analysis of the Jacobian matrix $\partial f(x)$ is thus useful to select $p$ and $\lambda$ in dominance analysis. The uniform splitting of the spectrum is necessary but of course not sufficient for dominance. This is well-known even for $p=0$. For instance, classical counterexamples to Kalman's conjecture [25] illustrate that a system can fail to be contractive even when the spectrum of its Jacobian is uniformly in the left half complex plane.

\section{B. Convex relaxations}

Sufficient conditions for dominance are provided by the inequality

$$
\partial f(x)^{T} P+P \partial f(x)+2 \lambda P+\varepsilon I \leq 0 \quad \forall x \in \mathcal{X}
$$

whose solutions $P$, for some $\varepsilon \geq 0$, must also satisfy a fixed inertia constraint.

If the spectrum of $\partial f(x)$ admits a stable splitting for a given $\lambda$, then all the solutions $P$ of 26 must share the same inertia, meaning that the inertia condition can be dropped. One is then left with solving an infinite family of LMIs.

It is common practice to reduce an infinite family of LMIS to a finite family through convex relaxation, see e.g. [4] and references therein. Let $\mathcal{A}:=\left\{A_{1}, \ldots, A_{N}\right\}$ be a family of matrices such that $\partial f(x) \in \operatorname{ConvexHull}(\mathcal{A})$ for all $x$. Then, by construction, any (uniform) solution $P$ to

$$
A_{i}^{T} P+P A_{i}+2 \lambda P+\varepsilon I \leq 0 \quad 1 \leq i \leq N
$$

is a solution to 26. For instance, at each $x$, $\partial f(x)=\sum_{i=1}^{N} \rho_{i}(x) A_{i}$ for a given set of $\rho_{i}(x)$ such that $\sum_{i=1}^{N} \rho_{i}(x)=1$. Thus, the left-hand side of 26) reads $\left(\sum_{i=1}^{N} \rho_{i}(x) A_{i}^{T}\right) P+P\left(\sum_{i=1}^{N} \rho_{i}(x) A_{i}\right)+2 \lambda P+\varepsilon I=$ $\sum_{i=1}^{N} \rho_{i}(x)\left(A_{i}^{T} P+P A_{i}+2 \lambda P+\varepsilon I\right) \leq 0$, where the last inequality follows from 27.

The algorithmic steps of dominance analysis of a given nonlinear system $\dot{x}=f(x)$ can thus be summarized as follows:

1) Estimate $p$ and $\lambda$ from the spectrum analysis of $\partial f(x)$

2) Reduce the infinite family of LMIs to a finite family by convex relaxations.

3) Test the feasibility of the relaxed LMI with a LMI solver.

\section{Example}

We illustrate the theory on a classical textbook example: a one degree of freedom mechanical system with nonlinear spring (Duffing model), actuated by a DC motor with a PI feedback control. While this example is elementary, it illustrates the tractability of dominance analysis on a fourdimensional model, for which a global analysis of the attractors is a nontrivial problem.
The mechanical model is given by

$$
\dot{x}_{p}=x_{v} \quad \dot{x}_{v}=-\alpha\left(x_{p}\right)-c x_{v}+u
$$

where $x_{p}$ and $x_{v}$ are position and velocity of the mass respectively, $u$ is the force input to the system, $c$ is the damping coefficient, and $\alpha\left(x_{p}\right):=\partial U\left(x_{p}\right)$ is the force deriving from the mechanical potential $U: \mathbb{R} \rightarrow \mathbb{R}$. Contraction, or 0dominance is expected with sufficient damping if the potential is strictly convex. A differential quadratic storage is easily found for the numerical value $c=5$ and the assumption

$$
1 \leq \partial \alpha\left(x_{p}\right) \leq 5
$$

$P$ is computed via convex relaxation (27) for $A_{1}:=\left[\begin{array}{cc}0 & 1 \\ -1 & -5\end{array}\right]$ and $A_{2}:=\left[\begin{array}{cc}0 & 1 \\ -5 & -5\end{array}\right]$. For $\lambda=0$ and $\varepsilon=0.01$, the LMI solver (Yalmip [27], SeDuMi [49] ) returns

$$
P:=\left[\begin{array}{ll}
0.8696 & 0.1482 \\
0.1482 & 0.1304
\end{array}\right]
$$

which is positive definite.

The same approach is repeated for the non-convex potential

$$
-2 \leq \partial \alpha\left(x_{p}\right) \leq 5,
$$

which allows for several minima (including the classical double-well potential of the nonlinear Duffing model [19. Chapter 2]). Figure 2 (left) suggests a stable splitting between the two eigenvalues. The differential storage is computed again by convex relaxation (27) for $A_{1}:=\left[\begin{array}{cc}0 & 1 \\ 2 & -5\end{array}\right]$ and $A_{2}:=\left[\begin{array}{cc}0 & 1 \\ -5 & -5\end{array}\right]$. For $\lambda=2$ and $\varepsilon=0.01$, the LMI solver returns

$$
P=\left[\begin{array}{cc}
-5.1987 & 3.6260 \\
3.6260 & 6.1987
\end{array}\right]
$$

which has inertia 1. Figure 2 (right) shows the non positive level sets of $\delta x^{T} P \delta x$. The system is 1-dominant, meaning that every bounded trajectory asymptotically converges to some fixed point.
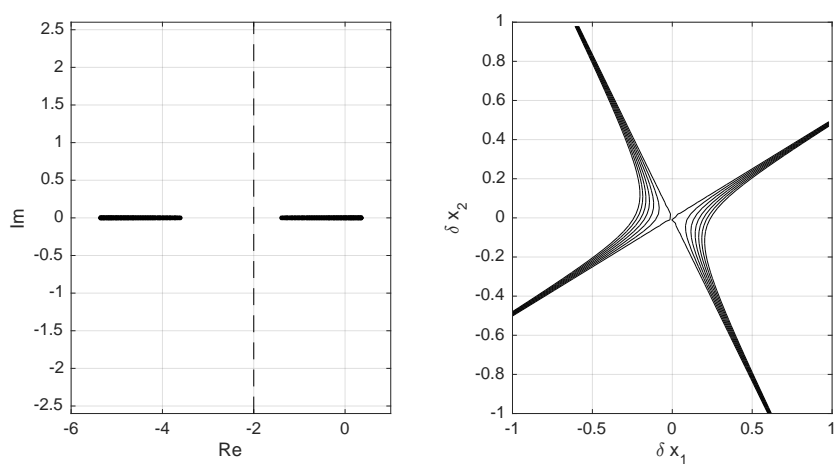

Figure 2. Left: roots of the Jacobian of the mechanical systems for different $x_{p}$. The value of $\lambda$ is emphasized by the vertical dashed line. Right: negative level sets of $\delta x^{T} P \delta x$ where $P$ has inertia 1 .

Suppose now that the mechanical system is driven by a DC motor modelled by the electrical equation

$$
u=k_{f} x_{i} \quad L \dot{x}_{i}=-R x_{i}-k_{e} x_{v}+V .
$$

$x_{i}$ is the current of the circuit, $k_{f}$ is a static approximation of the current to force characteristic, $L$ and $R$ are inductance 
and resistance respectively, $k_{e}$ is the back electromotive force coefficient, and the voltage $V$ is an additional input.

It is easy to verify that 1 -dominance is preserved for $R=1$, $k_{f}=1, k_{e}=1$, and $0<L<0.05$, since the time-scale separation between electrical and mechanical dynamics introduces a mild perturbation on the dominant/slow dynamics of the system. For $L=0.1$ the reduced time scale separation allows for interaction between electrical and mechanical dynamics. The distribution of the eigenvalues of the Jacobian in Figure 3 (left) suggests that strict 1-dominance still holds. Indeed, for $L=0.1, \lambda=2$, and $\varepsilon=0.01$, the LMI solver returns

$$
P=\left[\begin{array}{ccc}
-3.0942 & 0.8985 & -0.5355 \\
0.8985 & 3.3771 & 0.1935 \\
-0.5355 & 0.1935 & 0.7171
\end{array}\right]
$$

which has inertia 1 . For constant inputs $V$, every bounded trajectory necessarily converges to some fixed point, as illustrated in Figure 3 (right), where we considered the double well potential $U\left(x_{p}\right):=x_{p}^{2} / 4+\cos \left(x_{p}\right)$.
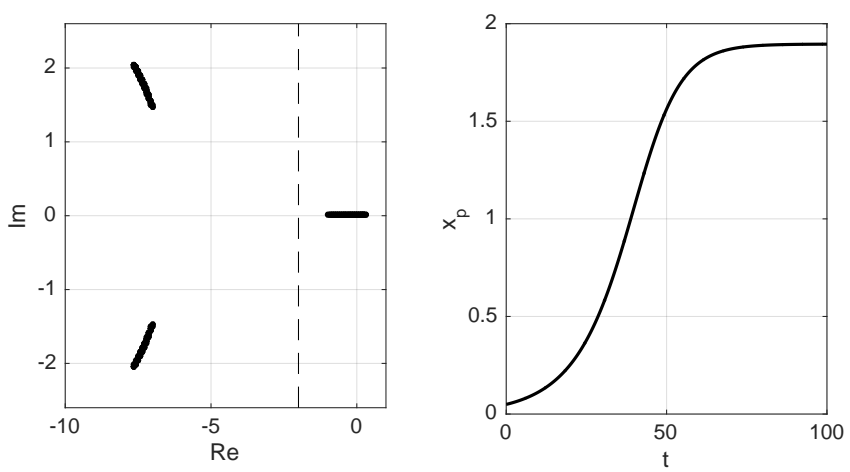

Figure 3. Left: roots of the Jacobian of (28) and 29], by sampling $-2 \leq$ $\partial \alpha\left(x_{p}\right) \leq 5$ at different $x_{p}$. Right: Trajectory of the mass position in time for the interconnected system [28, [29] with potential $U\left(x_{p}\right):=x_{p}^{2} / 4+$ $\cos \left(x_{p}\right)$, from the initial condition $x_{p}=0.05, x_{v}=0, x_{i}=0$, at constant $V=0$.

Finally, we close the loop with a PI controller

$$
V=k_{P}\left(r-x_{p}\right)+k_{I} x_{c} \quad \dot{x}_{c}=r-x_{p}
$$

where $x_{c}$ is the integrator variable, $k_{P}$ and $k_{I}$ are proportional and integral gains, respectively, and $r$ is the reference.

The degree of dominance of the closed loop can be modulated via PI control. A detailed analysis of PI control for $p$ dominance is beyond the scope of this paper. We just observe that with gains $k_{P}=1$ and $k_{I}=5$ the eigenvalues of the Jacobian in Figure 4 (left) exhibits a stable splitting into two groups of two eigenvalues. 2-dominance is verified with $\lambda=2$, $\varepsilon=0.01$, in which case the LMI solver returns the storage

$$
P=\left[\begin{array}{cccc}
-4.3713 & 1.7901 & -0.5507 & 0.0216 \\
1.7901 & 5.6483 & 0.3768 & -0.9320 \\
-0.5507 & 0.3768 & 1.0521 & -0.4363 \\
0.0216 & -0.9320 & -0.4363 & -1.3291
\end{array}\right]
$$

with inertia 2. For $r=0$ the unique fixed point at 0 is unstable. We conclude that every bounded trajectory must converge to a periodic orbit, as illustrated in Figure 4 (right).
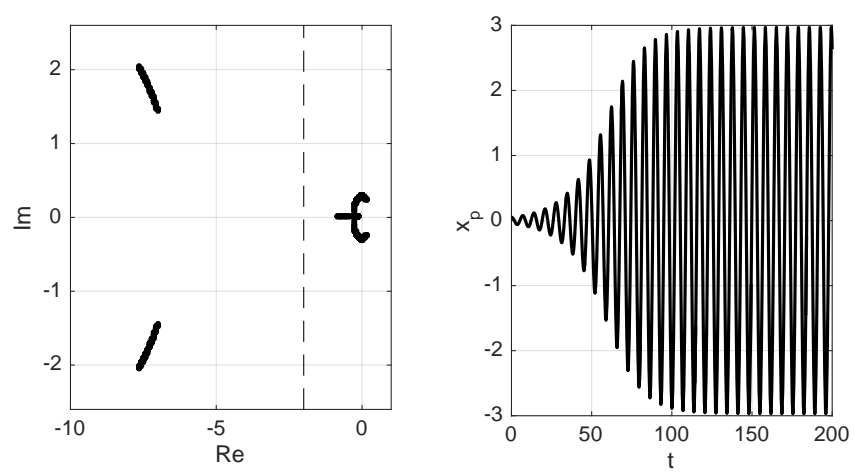

Figure 4. Left: roots of the Jacobian of the closed loop given by 28, 29 and 30 by sampling $-2 \leq \partial \alpha\left(x_{p}\right) \leq 5$ at different $x_{p}$. Right: Trajectory of the mass position in time for the closed loop [28, [29], 30] with potential $U\left(x_{p}\right):=x_{p}^{2} / 4+\cos \left(x_{p}\right)$ and PI control gains $k_{P}=1$ and $k_{I}=5$, from the initial condition $x_{p}=0.05, x_{v}=0, x_{i}=0$ and $x_{c}=0$, at constant $r=0$.

Remark 1: A variant of the example is to replace the mechanical model with the nonlinear pendulum $\dot{x}_{p}=x_{v}$, $\dot{x}_{v}=-\sin \left(x_{p}\right)-c x_{v}+u$ with $\left(x_{p}, x_{v}\right) \in \mathbb{S} \times \mathbb{R}$. Onedominance is proven as in Duffing example but the statespace is now nonlinear. From Theorem 1] every attractor of the pendulum exhibits at least one direction of contraction, locally. The shape of $\mathbb{S} \times \mathbb{R}$ makes 1-dominance compatible with fixed point attractors, for small constant torque $u$, and with attractors defined by periodic orbits, for large constant torque $u$, [48, Section 8.5]. See also the analysis in [13] using differential positivity.

\section{INTERCONNECTIONS}

\section{A. Differential dissipativity theory}

Dissipativity theory is a fundamental complement to stability theory in systems and control [54], [55], [56]. Stability, and more generally, dominance, is the property of a closed system. Dissipativity is the property of an open system, i.e. a system with inputs and outputs. By decomposing a closed system as an interconnection of open subsystems, the search of quadratic storage for the analysis of the closed system is converted into the solution of linear matrix inequalities for the open subsystems.

The recent papers [11], [15], [51] have proposed to use dissipativity theory differentially in order to study contraction (i.e. 0 -dominance) via interconnections. We pursue this approach to study $p$-dominance. We restrict to open systems of the form

$$
\left\{\begin{array}{rl}
\dot{x} & =f(x)+B u \\
y & =C x+D u
\end{array} \quad x \in \mathcal{X},(y, u) \in \mathcal{W}\right.
$$

where $\mathcal{X}$ and $\mathcal{W}$ are smooth manifolds of dimension $n$ and $m$, respectively. The associated prolonged system reads

$$
\left\{\begin{aligned}
\dot{x} & =f(x)+B u \\
\dot{\delta} x & =\partial f(x) \delta x+B \delta u \\
y & =C x+D u \\
\delta y & =C \delta x+D \delta u
\end{aligned}\right.
$$

where $(x, \delta x) \in T \mathcal{X}$ and $(w, \delta w):=((y, u),(\delta y, \delta u)) \in T \mathcal{W}$. 
We also assume that $\mathcal{W}$ is covered by a single chart and that the matrix $L$ and the symmetric matrices $Q$ and $R$ below have suitable dimensions. All restrictions above relate to the restriction of constant tensors $P$ in this paper for the analysis of dominance.

Definition 3: A nonlinear system (31) is differentially $p$ dissipative with rate $\lambda$ and differential supply rate

$$
s(w, \delta w):=\left[\begin{array}{l}
\delta y \\
\delta u
\end{array}\right]^{T}\left[\begin{array}{cc}
Q & L \\
L^{T} & R
\end{array}\right]\left[\begin{array}{l}
\delta y \\
\delta u
\end{array}\right]
$$

if for some symmetric matrix $P$ with inertia $p$ and some constant $\varepsilon \geq 0$, the prolonged system (32) satisfies the conic constraint

$$
\left[\begin{array}{l}
\dot{\delta x} \\
\dot{\delta x}
\end{array}\right]^{T}\left[\begin{array}{cc}
0 & P \\
P & 2 \lambda P+\varepsilon I
\end{array}\right]\left[\begin{array}{l}
\dot{\delta} x \\
\delta x
\end{array}\right] \leq\left[\begin{array}{l}
\delta y \\
\delta u
\end{array}\right]^{T}\left[\begin{array}{cc}
Q & L \\
L^{T} & R
\end{array}\right]\left[\begin{array}{l}
\delta y \\
\delta u
\end{array}\right]
$$

for all $(x, \delta x) \in T \mathcal{X}$ and all $(w, \delta w) \in \mathcal{W}$. (31) is strictly differentially $p$-dissipative if $\varepsilon>0$.

Differential dissipativity is guaranteed by the feasibility of the inequality

$$
\left[\begin{array}{cc}
\partial f(x)^{T} P+P \partial f(x)-C^{T} Q C+2 \lambda P+\varepsilon I & P B-C^{T} L-C^{T} Q D \\
B^{T} P-L^{T} C-D^{T} Q C & -R-D^{T} L-L^{T} D-D^{T} Q D
\end{array}\right] \leq 0
$$

for some symmetric matrix $P$ with inertia $p$ and some constant $\varepsilon \geq 0$. A necessary condition for the feasibility of this inequality is that $\partial f(x)^{T} P+P \partial f(x)-C^{T} Q C+2 \lambda P+\varepsilon I \leq 0$ which corresponds to (26) for $Q=0$ and clarifies the connection between differential dissipativity and dominance. This infinite family of LMIs can reduced to a finite family through relaxations, following the approach in Section VI-B.

\section{B. A dissipativity theorem for $p$-dominance}

Suppose that a nonlinear system can be decomposed as the interconnection of two subsystems:

$$
\begin{aligned}
& \left\{\begin{array}{l}
\dot{x}_{1}=f_{1}\left(x_{1}\right)+B_{1} u_{1} \\
y_{1}=\underline{C} x_{1}+D_{1} u_{1}
\end{array}\right. \\
& \left\{\begin{array}{l}
\dot{x}_{2}=f_{2}\left(x_{2}\right)+B_{2} u_{2} \\
y_{2}=\bar{C} x_{2}+D_{2} u_{2}
\end{array}\right. \\
& u=H y+v
\end{aligned}
$$

where the matrix $H$ specifies the interconnection pattern between inputs $u=\left[\begin{array}{ll}u_{1}^{T} & u_{2}^{T}\end{array}\right]^{T}$ and outputs $y=\left[\begin{array}{ll}y_{1}^{T} & y_{2}^{T}\end{array}\right]^{T}$. $v=\left[\begin{array}{ll}v_{1}^{T} & v_{2}^{T}\end{array}\right]^{T}$ is an additional input. Then the dissipativity theorem below provides conditions for the differential $p$ dissipativity of the interconnected system from the differential $p$-dissipativity of its components.

The formulation of the theorem follows the approach of [33]. The theorem can be easily adapted to the interconnection of several systems [34]. In what follows we will use $\bar{Q}:=\left[\begin{array}{l|l}Q_{1} \mid & \\ & Q_{2}\end{array}\right], \bar{L}:=\left[\begin{array}{l|l}L_{1} & \\ \hline L_{2}\end{array}\right]$, and $\bar{R}:=\left[\begin{array}{l|l}R_{1} \mid & \\ \hline R_{2}\end{array}\right]$ for readability, where for $i \in\{1,2\}$ the matrices $Q_{i}, L_{i}$ and $R_{i}$ characterize the differential supply rate fo each subsystem.

Theorem 4: Let 35a and 35b be (strictly) differentially $p_{1}$-dissipative and $p_{2}$-dissipative respectively, with uniform rate $\lambda$ and differential supply rate

$$
\left[\begin{array}{c}
\delta y_{i} \\
\delta u_{i}
\end{array}\right]^{T}\left[\begin{array}{cc}
Q_{i} & L_{i} \\
L_{i}^{T} & R_{i}
\end{array}\right]\left[\begin{array}{c}
\delta y_{i} \\
\delta u_{i}
\end{array}\right] \quad i \in\{1,2\}
$$

Then, the interconnected system (35) is (strictly) differentially $p$-dissipative with degree $p=p_{1}+p_{2}$, rate $\lambda$, and differential supply rate

$$
\left[\begin{array}{l}
\delta y \\
\delta v
\end{array}\right]^{T}\left[\begin{array}{cc}
Q & L \\
L^{T} & R
\end{array}\right]\left[\begin{array}{l}
\delta y \\
\delta v
\end{array}\right]
$$

where

$$
\begin{aligned}
Q & :=\bar{Q}+\bar{L} H+H^{T} \bar{L}^{T}+H^{T} \bar{R} H \\
L & :=\bar{L}+H^{T} \bar{R} \\
R & :=\bar{R} .
\end{aligned}
$$

For $v=0$, the interconnected system (35) is (strictly) $p$ dominant if $Q \leq 0$.

Proof: Let $P_{1}$ and $P_{2}$ be solutions to (3) respectively for 35a and (35b). Take $P:=P_{1}+P_{2}, x:=\left[\begin{array}{ll}x_{1}^{T} & x_{2}^{T}\end{array}\right]^{T}$, and $\delta x:=\left[\begin{array}{ll}\delta x_{1}^{T} & \delta x_{2}^{T}\end{array}\right]^{T}$. Note that $P_{1}$ has inertia $p_{1}$ and $P_{2}$ has inertia $p_{2}$, where $n_{1}$ and $n_{2}$ are the dimensions of the two state manifolds, respectively. It follows that $P$ has inertia $p_{1}+p_{2}$. Furthermore, by (strict) differential dissipativity of 35a and 35b, (3) can be written in the aggregated form $\dot{\delta}^{T} P \delta x+\delta x^{T} P \dot{\delta} x+\delta x^{T}(2 \lambda P+\varepsilon I) \delta x \leq$ $\delta y^{T} \bar{Q} \delta y+\delta y^{T} \bar{L} \delta u+\delta u^{T} \bar{L}^{T} \delta y+\delta u^{T} \bar{R} \delta u$, for some $\varepsilon \geq$ $(\varepsilon>0)$. Since $\delta u=H \delta y+\delta v$, the expression above reads $\dot{\delta x^{T}} P \delta x+\delta x^{T} P \dot{\delta} x+\delta x^{T}(2 \lambda P+\varepsilon I) \delta x \leq \delta y^{T} Q \delta y+$ $\delta y^{T} L \delta v+\delta v^{T} L^{T} \delta y+\delta u^{T} R \delta u$ which shows (strict) differential $p$-dissipativity of the interconnected system.

Finally, for $v=0$ we get $\dot{\delta x}^{T} P \delta x+\delta x^{T} P \dot{\delta} x+\delta x^{T}(2 \lambda P+$ $\varepsilon I) \delta x \leq \delta y^{T} Q \delta y \leq 0$ where the last inequality follows from the condition $Q \leq 0$. This shows that $P$ is a solution of (12).

Theorem 4 is a general result to study dominance via interconnections. In particular, it provides an interconnection theorem for the analysis of contraction (0-dominance) and monotonicity (1-dominance).

\section{Differential passivity analysis}

The passivity supply

$$
\left[\begin{array}{l}
\delta y \\
\delta u
\end{array}\right]^{T}\left[\begin{array}{ll}
0 & I \\
I & 0
\end{array}\right]\left[\begin{array}{l}
\delta y \\
\delta u
\end{array}\right]
$$

plays an important role in dissipativity theory because it connects the theory to the physical property that a passive system can only store the energy supplied by its environment. The theory of port-Hamiltonian systems encompasses a broad modeling framework of physical models with passivity properties [52].

The passivity theorem states that the feedback interconnection of passive systems is passive. A differential version of this important theorem is provided by Theorem 4 any system that is differentially $p$-passive preserves that property under the feedback interconnection with a differentially passive (i.e. 0-passive) system.

Passivity theory has proven useful in identifying robust controller structures that preserve stability. An important particular case is the class of proportional-integral controllers, that we now revisit in the light of dominance analysis. 
We first consider the proportional feedback controller

$$
\text { (P) } \bar{y}=k_{P}(\bar{u}) \text {. }
$$

The controller is differentially 0-passive (for arbitrary nonnegative rate) from $\bar{u}$ to $\bar{y}$ provided that the mapping $k_{p}(\cdot)$ is monotone, or differentially positive: $\partial k_{P}(\bar{u}) \geq 0$ for all $\bar{u} \in \mathbb{R}$. For instance,

$$
\left[\begin{array}{l}
\delta \bar{y} \\
\delta \bar{u}
\end{array}\right]^{T}\left[\begin{array}{ll}
0 & I \\
I & 0
\end{array}\right]\left[\begin{array}{l}
\delta \bar{y} \\
\delta \bar{u}
\end{array}\right]=2 \delta \bar{u}^{T} \partial k(\bar{u}) \delta \bar{u} \geq 0 .
$$

Likewise, the proportional-integral feedback controller

$$
(P I) \quad \dot{x}_{c}=\bar{u}, \bar{y}=k_{P}(\bar{u})+k_{I} x_{c}
$$

from $\bar{u}$ to $\bar{y}$ is

- differentially 0-passive if $k_{P}(\cdot)$ is monotone and if $k_{I} \geq$ 0 , with rate $\lambda=0$.

- differentially 1 -passive if $k_{P}(\cdot)$ is monotone and if $k_{I}<$ 0 , with rate $\lambda \geq 0$ (strictly for $\lambda>0$ ).

This is because the storage $S\left(\delta x_{c}\right):=\frac{k_{I}}{2} \delta x_{c}^{2}$ satisfies $\dot{S} \leq$ $\delta \bar{u} \delta \bar{y}$. Furthermore, for $\lambda>0$ and $k_{I}<0$ we have $\dot{S}+$ $\lambda k_{I} \delta x_{c}^{2}+\varepsilon \delta x_{c}^{2} \leq \delta \bar{u} \delta \bar{y}$ for $\varepsilon=\lambda\left|k_{I}\right|$.

As an illustration, we revisit the nonlinear (Duffing) massspring-damper system in Section VI-C. For any nonlinear spring satisfying $-3 \leq \partial \alpha\left(x_{p}\right) \leq 3$, the system is strictly differential 1-passive with rate $\lambda=2$ from $u$ to $y=-x_{p}$ : defining the state $x:=\left[\begin{array}{ll}x_{p} & x_{v}\end{array}\right]$, the variational dynamics $\dot{\delta} x=A(x) \delta x+B \delta u, \delta y=C \delta x$

$$
A(x):=\left[\begin{array}{cc}
0 & 1 \\
-\partial \alpha\left(x_{p}\right) & -5
\end{array}\right] \quad B:=\left[\begin{array}{l}
0 \\
1
\end{array}\right] \quad C:=\left[\begin{array}{ll}
-1 & 0
\end{array}\right] .
$$

satisfies

$$
\begin{aligned}
A(x)^{T} P+P A(x)+2 \lambda P+\varepsilon I & \leq 0 \\
P B & =C^{T}
\end{aligned}
$$

for $P=\left[\begin{array}{cc}-2 & -1 \\ -1 & 0\end{array}\right]$ and $\varepsilon=0.01$, for all $x \in \mathbb{R}^{2}$.

Assume for simplicity that $k_{P}(\cdot)$ is odd and monotone and consider the interconnection

$$
u=\bar{y}+v \quad \bar{u}=-y .
$$

The closed loops with the proportional controller and with the proportional-integral controller are illustrated in Figure 5 for the the particular case $k_{P}(\bar{u}):=\tanh (2 \bar{u})$ and $k_{I}:=-1$.

By Theorem 4 the proportional feedback $u=-k_{P}(y)+$ $v=k_{P}\left(x_{p}\right)+v$ preserves 1-differential passivity. Figure 6 (left) illustrates a situation where the original system is monostable (linear spring, i.e. a quadratic potential) and then converted to a bistable system with the static output feedback $u=-\tanh (2 y)+v=\tanh \left(2 x_{p}\right)+v$. The example is elementary but illustrative of a general principle: the feedback controller shapes the potential energy of a contractive mechanical system to convert the system from monostable to bistable.

Likewise, the proportional-integral control $u=-k_{P}(y)-$ $k_{I} \int y+v=k_{P}\left(x_{p}\right)-k_{I} \int y+v=\tanh \left(2 x_{p}\right)-\int x_{p}+v$ makes the closed-loop system differentially 2-passive because it corresponds to the negative feedback interconnection of two differentially 1 -passive systems. Figure 7 illustrates that, in this new configuration, the role of the proportional controller is to convert a linear stable system $\left(k_{P}=0\right)$ into a 2-dominant system with a stable limit cycle oscillation $\left(k_{P}=1\right)$.

Again the example is elementary but illustrates the general principle that PI control can turn a contractive mechanical system into a system with a limit cycle attractor. Such conclusions are usually drawn from a local analysis of the linearization around the stable equilibrium of the contractive system. The first scenario corresponds to a saddle node bifurcation whereas the second scenario corresponds to a Hopf bifurcation. The differential approach in this paper makes this analysis non local.
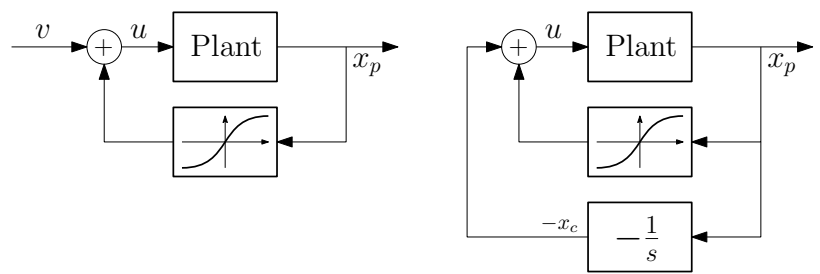

Figure 5. Left: Closed loop of the plant given by 28) with $\alpha\left(x_{p}\right)=x_{p}$ and $u=\tanh \left(2 x_{p}\right)+v$ (saturated proportional feedback). Right: Closed loop given by the interconnection of the linear mass-spring-damper system and nonlinear proportional-integral controller.
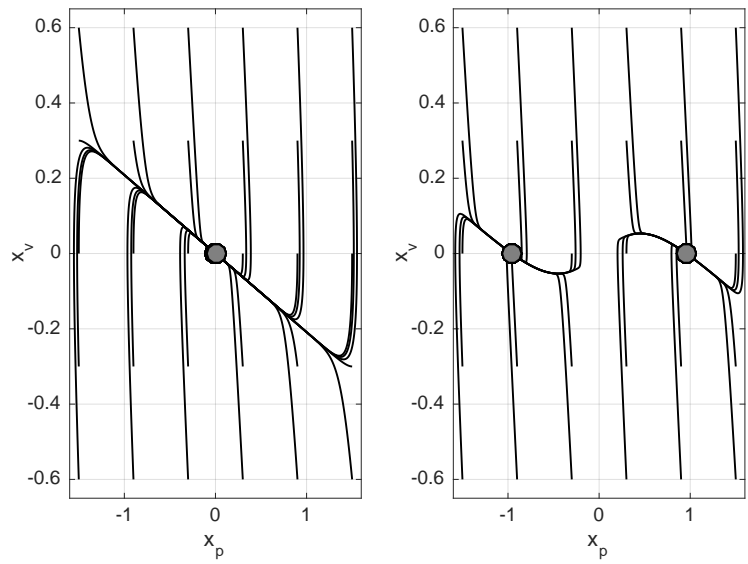

Figure 6. Left: trajectories from different initial conditions of the open loop system 28 with $\alpha\left(x_{p}\right)=x_{p}$ and $u=0$. Right: trajectories from different initial conditions of the closed loop [28, $u=\tanh \left(2 x_{p}\right)+v$, with $\alpha\left(x_{p}\right)=x_{p}$ and $v=0$.

\section{Differential small gain analysis}

The supply

$$
\left[\begin{array}{l}
\delta y \\
\delta u
\end{array}\right]^{T}\left[\begin{array}{cc}
-I & 0 \\
0 & \gamma^{2} I
\end{array}\right]\left[\begin{array}{l}
\delta y \\
\delta u
\end{array}\right]
$$

also has a special status in dissipativity theory because of its connection to the small gain theorem, a cornerstone of robust control theory [58], [57], [9], [59], [50].

When specialized to the supply (42, Theorem 4 provides a differential version of the small gain theorem: $p$-dominance is preserved under feedback with a 0 -dominant system provided 

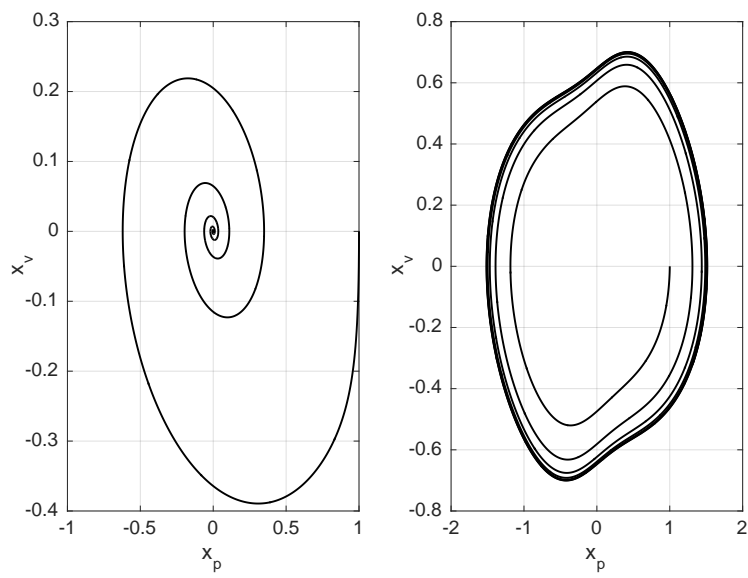

Figure 7. Left: Trajectory of the linear closed-loop system $\left(k_{P}=0\right)$. Right: Trajectory of the nonlinear closed-loop system $\left(k_{P}=1\right)$. Parameters: $\alpha(x)=x_{p}$. Initial condition $x_{p}=1, x_{v}=0, x_{c}=0$.

that their finite differential gains $\gamma_{1}$ and $\gamma_{2}$ (of degree $p$ and 0 respectively) satisfy the small gain condition $\gamma_{1} \gamma_{2}<1$.

The differential small gain theorem opens the way to a differential approach of nonlinear robust control. The link with an operator-theoretic definition of the differential gain of a dominant system is beyond the scope of this paper (see e.g. [17] for a concept of differential gain for stable systems), but we briefly illustrate how the theorem can be used in nonlinear robustness analysis.

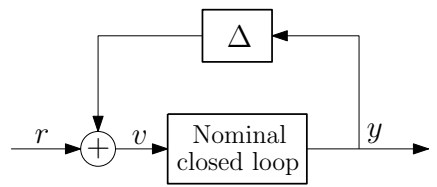

Figure 8. A nonlinear plant with parametric uncertainties represented as the feedback interconnection of nominal plant and uncertain dynamics $\Delta$.

Consider the nominal closed loop in Figure 5 (right) where the mass-spring-damper system 28 with linear spring $\alpha\left(x_{p}\right)=x_{p}$ is interconnected to a saturated proportionalintegral feedback $u=\tanh \left(2 x_{p}\right)-\int x_{p}+v$. We study the robustness of the oscillations to perturbations affecting the spring constant and damping coefficient. We use the usual representation of parametric uncertainties through feedback interconnections as shown in Figure 8

For $c=5$ and $\lambda=2$ the nominal closed loop system is 2-dominant with differential gain $\gamma:=0.5636$ from the input $v$ to the position output $x_{p}$, obtained for

$$
P:=\left[\begin{array}{ccc}
-0.5522 & 0.0498 & -0.0171 \\
0.0498 & 1.4946 & 0.3068 \\
-0.0171 & 0.3068 & 0.0576
\end{array}\right] .
$$

The nonlinear mechanical spring is modeled through an additive perturbation, i.e.

$$
\alpha\left(x_{p}\right)=x_{p}+\Delta\left(x_{p}\right),
$$

corresponding to the feedback interconnection in Figure 8 . with $v=\Delta\left(x_{p}\right)$. The differential small-gain theorem implies that 2-dominance is preserved for any $\left|\partial \Delta\left(x_{p}\right)\right| \leq 1 / \gamma$. Furthermore, for any perturbation $\Delta\left(x_{p}\right)$ that preserves the origin as a unique and unstable fixed point of the closed loop system, every bounded trajectory of the perturbed closed loop will converge to a periodic orbit, like in the nominal case.

The analysis of perturbations affecting the damping coefficient $c=5$ is similar. For $c=5$ and $\lambda=2$ the nominal closed loop system is 2-dominant with differential gain $\gamma:=0.5468$ from the input $v$ to the velocity output $x_{v}$, obtained for

$$
P:=\left[\begin{array}{ccc}
-0.2859 & -0.0028 & -0.0131 \\
-0.0028 & 1.2328 & 0.2977 \\
-0.0131 & 0.2977 & 0.0532
\end{array}\right] .
$$

Oscillations will persist when the linear damping coefficient $c x_{v}$ is replaced by a nonlinear coefficient $c\left(x_{v}\right)$ given by

$$
c\left(x_{v}\right)=5 x_{v}+\Delta\left(x_{v}\right)
$$

provided that $\left|\partial \Delta\left(x_{v}\right)\right|<1 / \gamma$ and that the origin remains an unstable fixed point.

\section{CONCLUSION}

This paper illustrated that linear-quadratic dissipativity theory, a cornerstone of stability theory, generalizes with surprising ease to the analysis of dominance. Dominance analysis in turn is relevant to capture the frequent property that the asymptotic behavior of a nonlinear dynamical model is lowdimensional. In particular, the theory seems relevant to generalize the theory of stability to a theory of multistability and limit cycle analysis.

The approach in this paper is differential, meaning that the usual linear matrix inequalities of dissipativity theory are considered in the tangent bundle. They characterize a dominated splitting of the linearized flow between $p$ dominant directions and $n-p$ transient directions. This property is captured with the usual linear matrix inequalities of dissipativity theory, with the only difference that the solution matrix $P$ is required to have a fixed inertia ( $p$ negative eigenvalues and $n-p$ positive eigenvalues), the standard stability framework corresponding to the case $p=0$.

An important restriction throughout this paper is to analyze $p$-dominance with a constant quadratic storage (i.e. a constant $P)$. This restriction is the price to be paid for tractability. Standard LMI solvers can then be used to construct the storage.

A number of generalizations deserve further attention. Those include the construction of differential storages that are non quadratic, or/and state-dependent (i.e. non constant $P(x)$ ), as well as the study of dominance with state-dependent rate $\lambda(x)$, or systems with a different degree of dominance in different parts of the state-space, or the analysis of dominance in non smooth systems. Such generalizations have received considerable attention in the analysis of contraction, i.e. 0dominance, suggesting clear avenues to study $p$-dominance. Finally, differential dissipativity theory offers an opportunity to revisit classical results from robust control theory and absolute stability theory in the context of multistable and oscillatory systems, see e.g. [30] for a first step in that direction. 


\section{REFERENCES}

[1] D. Angeli and E.D. Sontag. Monotone control systems. IEEE Transactions on Automatic Control, 48(10):1684 - 1698, 2003.

[2] A. Katok B. Hasselblatt. Handbook of dynamical systems, volume 1A. Elsevier Science, first edition, 2002.

[3] S. Bonnabel, P. Martin, and P. Rouchon. Non-linear symmetrypreserving observers on Lie groups. IEEE Transactions on Automatic Control, 54(7):1709-1713, July 2009.

[4] S. Boyd, L. El Ghaoui, E. Feron, and V. Balakrishnan. Linear Matrix Inequalities in System and Control Theory. SIAM, 1994.

[5] P.J. Bushell. Hilbert's metric and positive contraction mappings in a Banach space. Archive for Rational Mechanics and Analysis, 52(4):330 338,1973

[6] F. Colonius, L. Grüne, and W. Kliemann. The Dynamics of Control. Systems \& Control: Foundations \& Applications. Birkhäuser Boston, 2012.

[7] P.E. Crouch and A.J. van der Schaft. Variational and Hamiltonian control systems. Lecture notes in control and information sciences. Springer, 1987.

[8] S. Crovisier and R. Potrie. Introduction to partial hyperbolic dynamics. Technical report, Minicourse, 2015. Minicourse.

[9] C.A. Desoer and M. Vidyasagar. Feedback Systems: Input-Output Properties, volume 55 of Classics in Applied Mathematics. Society for Industrial and Applied Mathematics, 1975.

[10] F Forni. Differential positivity on compact sets. In 54th IEEE Conference on Decision and Control, pages 6355-6360, 2015.

[11] F. Forni and R. Sepulchre. On differentially dissipative dynamical systems. In 9th IFAC Symposium on Nonlinear Control Systems, 2013.

[12] F. Forni and R. Sepulchre. A differential Lyapunov framework for contraction analysis. IEEE Transactions on Automatic Control, 59(3):614$628,2014$.

[13] F. Forni and R. Sepulchre. Differentially positive systems. IEEE Transactions on Automatic Control, 61(2):346-359, 2016.

[14] F. Forni and R. Sepulchre. A dissipativity theorem for p-dominant systems. In 56th IEEE Conference on Decision and Control, 2017.

[15] F. Forni, R. Sepulchre, and A.J. van der Schaft. On differential passivity of physical systems. In 52nd IEEE Conference on Decision and Control, 2013.

[16] V. Fromion and G. Scorletti. Connecting nonlinear incremental Lyapunov stability with the linearizations Lyapunov stability. In 44th IEEE Conference on Decision and Control, pages 4736 - 4741, December 2005.

[17] T.T. Georgiou. Differential stability and robust control of nonlinear systems. Mathematics of Control, Signals and Systems, 6(4):289-306, 1993.

[18] C. Grussler and A. Rantzer. Modified balanced truncation preserving ellipsoidal cone-invariance. In 53rd IEEE Conference on Decision and Control, pages 2365-2370, Dec 2014.

[19] J. Guckenheimer and P. Holmes. Nonlinear oscillations, dynamical systems, and bifurcations of vector fields. Springer-Verlag, New York, Berlin, Heidelberg, Tokyo, page 0, 1986.

[20] M.W. Hirsch. Stability and convergence in strongly monotone dynamical systems. Journal für die reine und angewandte Mathematik, 383:1-53, 1988.

[21] M.W. Hirsch, C.C. Pugh, and M. Shub. Invariant Manifolds. Lecture Notes in Mathematics 583. Springer Berlin Heidelberg, 1977.

[22] M.W. Hirsch and S. Smale. Differential Equations, Dynamical Systems, and Linear Algebra (Pure and Applied Mathematics, Vol. 60). Academic Press, 1974.

[23] M.W. Hirsch and H.L. Smith. Competitive and cooperative systems: A mini-review. In L. Benvenuti, A. Santis, and L. Farina, editors, Positive Systems, volume 294 of Lecture Notes in Control and Information Science, pages 183-190. Springer Berlin Heidelberg, 2003.

[24] M.W. Hirsch and H.L. Smith. Monotone dynamical systems. In P. Drabek A. Canada and A. Fonda, editors, Handbook of Differential Equations: Ordinary Differential Equations, volume 2, pages 239 - 357. North-Holland, 2006.

[25] R.E. Kalman. Physical and mathematical mechanisms of instability in nonlinear automatic control systems. Transactions of ASME, 79(3):553566,1957

[26] A. Katok and B. Hasselblatt. Introduction to the Modern Theory of Dynamical Systems. Encyclopedia of Mathematics and its Applications. Cambridge University Press, 1997.

[27] J. Lofberg. Yalmip : a toolbox for modeling and optimization in Matlab. In IEEE International Symposium on Computer Aided Control Systems Design, pages 284-289, 2004.

[28] W. Lohmiller and J.E. Slotine. On contraction analysis for non-linear systems. Automatica, 34(6):683-696, June 1998.

[29] D.G. Luenberger. Introduction to Dynamic Systems: Theory, Models, and Applications. Wiley, 1 edition, 1979.

[30] F.A. Miranda-Villatoro, F. Forni, and R. Sepulchre. Analysis of Lur'e dominant systems in the frequency domain. Submitted to Automatica, https://arxiv.org/abs/1710.01645, 2017.
[31] C. Mostajeran and R. Sepulchre. Differential positivity with respect to cones of rank k. IFAC-PapersOnLine, 50(1):7439 - 7444, 2017. 20th IFAC World Congress.

[32] C. Mostajeran and R. Sepulchre. Positivity, monotonicity, and consensus on lie groups. To appear in SIAM Journal of Control and Optimization, 2018.

[33] P. Moylan. Dissipative systems and stability. Lecture Notes in collaboration with D. Hill, University of Newcastle, www.pmoylan.org, 2014.

[34] P. Moylan and D. Hill. Tests for stability and instability of interconnected systems. IEEE Transactions on Automatic Control, 24(4):574-575, 1979.

[35] S. Newhouse. Cone-fields, domination, and hyperbolicity. In M. Brin, B. Hasselblatt, A.B. Katok, and Y. Pesin, editors, Modern Dynamical Systems and Applications, pages 419-432. Cambridge University Press, 2004.

[36] A. Pavlov, N. van de Wouw, and H. Nijmeijer. Uniform Output Regulation of Nonlinear Systems: A Convergent Dynamics Approach. Systems \& Control: Foundations \& Applications. Birkhäuser, 2005

[37] Y.B. Pesin. Lectures on Partial Hyperbolicity and Stable Ergodicity. Zurich lectures in advanced mathematics. European Mathematical Society, 2004.

[38] J.F. Plante. Anosov flows. American Journal of Mathematics, 94(3):pp. 729-754, 1972

[39] G. Russo, M. Di Bernardo, and E.D. Sontag. Global entrainment of transcriptional systems to periodic inputs. PLoS Computational Biology, 6(4):e1000739, 042010.

[40] M. Sambarino. A (short) survey on Dominated Splitting. ArXiv e-prints, 2014.

[41] L.A. Sanchez. Cones of rank 2 and the Poincaré-Bendixson property for a new class of monotone systems. Journal of Differential Equations, 246(5): 1978 - 1990, 2009.

[42] H.L. Smith. Monotone Dynamical Systems: An Introduction to the Theory of Competitive and Cooperative Systems, volume 41 of Mathematical Surveys and Monographs. American Mathematical Society, 1995.

[43] R.A. Smith. Existence of period orbits of autonomous ordinary differential equations. In Proceedings of the Royal Society of Edinburgh, volume 85A, pages 153-172, 1980.

[44] R.A. Smith. Orbital stability for ordinary differential equations. Journal of Differential Equations, 69(2):265 - 287, 1987.

[45] E.D. Sontag. Contractive systems with inputs. In Jan C. Willems, Shinji Hara, Yoshito Ohta, and Hisaya Fujioka, editors, Perspectives in Mathematical System Theory, Control, and Signal Processing: A Festschrift in Honor of Yutaka Yamamoto on the Occasion of his 60th Birthday, pages 217-228. Springer Berlin Heidelberg, Berlin, Heidelberg, 2010.

[46] R.J. Stern and Wolkowiczm H. Invariant ellipsoidal cones. Linear Algebra and its Applications, 150:81 - 106, 1991.

[47] R.J. Stern and H. Wolkowicz. Exponential nonnegativity on the ice cream cone. SIAM Journal on Matrix Analysis and Applications. 12(1):160-165, 1991.

[48] S.H. Strogatz. Nonlinear Dynamics And Chaos. Westview Press, 1994.

[49] J.F. Sturm. Using SeDuMi 1.02, a MATLAB toolbox for optimization over symmetric cones. Optimization Methods and Software, 11-12:625653, 1999.

[50] A.J. van der Schaft. $L_{2}$-Gain and Passivity in Nonlinear Control. Springer-Verlag New York, Inc., Secaucus, N.J., USA, second edition, 1999.

[51] A.J. van der Schaft. On differential passivity. In 9th IFAC Symposium on Nonlinear Control Systems, 2013.

[52] A.J. van der Schaft and D. Jeltsema. Port-hamiltonian systems theory:

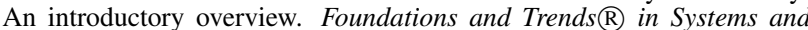
Control, 1(2-3):173-378, 2014.

[53] S. Wiggins. Normally Hyperbolic Invariant Manifolds in Dynamical Systems. Applied Mathematical Sciences. Springer, 1994.

[54] J.C. Willems. Dissipative dynamical systems part I: General theory. Archive for Rational Mechanics and Analysis, 45:321-351, 1972.

[55] J.C. Willems. Dissipative dynamical systems part II: Linear systems with quadratic supply rates. Archive for Rational Mechanics and Analysis, 45:352-393, 1972.

[56] J.C. Willems. Dissipative dynamical systems. European Journal of Control, 13(2-3):134-151, 2007.

[57] G. Zames. On the input-output stability of time-varying nonlinear feedback systems-part II: Conditions involving circles in the frequency plane and sector nonlinearities. IEEE Transactions on Automatic Control, 11(3):465-476, 1966.

[58] G. Zames. On the input-output stability of time-varying nonlinear feedback systems part I: Conditions derived using concepts of loop gain, conicity, and positivity. IEEE Transactions on Automatic Control, 11(2):228-238, 1966

[59] K. Zhou, J.C. Doyle, and K. Glover. Robust and optimal control. Prentice Hall, 1995 